\title{
Complements of Subanalytic Sets and Existential Formulas for Analytic Functions
}

\author{
Andrei Gabrielov \\ Department of Mathematics, Purdue University \\ West Lafayette, IN 47907-1395, USA \\ e-mail: agabriel@math.purdue.edu
}

Received August 6, 1995

\begin{abstract}
We show that the complement of a subanalytic set defined by real analytic functions from any subalgebra closed under differentiation is a subanalytic set defined by the functions from the same subalgebra. This result has an equivalent formulation in logic: Consider an expression built from functions as above using equalities and inequalities as well as existential and universal quantifiers. Such an expression is equivalent to an existential expression involving functions from the same class, provided that the variables approach neither infinity nor the boundary of the domain.
\end{abstract}

1. Introduction. The class of subanalytic sets was first considered by Gabrielov [8, $9]$ as a natural extension of the Tarski-Seidenberg $[22,24]$ theorem on elimination of quantifiers from semialgebraic expressions and of Łojasiewicz's [19, 20] theory of semianalytic sets. It was studied by Hironaka [16], where the term "subanalytic" was introduced, and by other authors $[1,2,4-6,13-15,21,23,25]$.

Subanalytic sets are defined as images of relatively proper real analytic maps of semianalytic sets. The main theorem of [8] asserts that the complement of any subanalytic set is subanalytic. This geometric property is equivalent to "quantifier simplification" i.e., the possibility to replace an expression involving real analytic equalities and inequalities, with existential and universal quantifiers, by an equivalent existential expression, provided that the variables are bounded and never approach the boundary of the domain of definition of the functions.

In $[4,6]$ this property is treated, within the framework of mathematical logic, as model completeness and $o$-minimality of the extension of the field of real numbers by "restricted" real analytic functions. The term "restricted" replaces the compactness conditions. It means that the functions are defined and analytic in the closed unit cube $[0,1]^{n}$ in $\mathbf{R}^{n}$, and extended by zero outside the cube. The result in [4] is even stronger: any expression involving equalities and inequalities with restricted analytic functions is equivalent to an expression without quantifiers when we allow "bounded division" of restricted analytic functions, i.e., for any two functions $P$ and $Q$, consider also $P / Q$ restricted to $\{P<Q\}$. 
The natural question of whether quantifier simplification is possible for special classes of analytic functions was treated in [26] for Pfaffian functions.

In this paper, we show that, for a subanalytic set defined by real analytic functions from a set $\Phi$, its complement (within the unit cube) is again a subanalytic set, defined by functions from the algebra $A$ generated by the functions from $\Phi$, their partial derivatives, constants 0 and 1 , and coordinate functions. In terms of mathematical logic, this means that any formula with functions from $A$ and with universal and existential quantifiers, is equivalent to an existential formula with the functions from $A$.

The proof is purely geometric. It is based on the finiteness properties of semianalytic sets.

As already shown in [8], quantifier simplification immediately implies various geometric properties of subanalytic sets (for example, Lojasiewicz inequality and the existence of Whitney stratifications) in the same way that the Tarski-Seidenberg theorem implies these properties for semialgebraic sets.

Some of our results are new also for the semianalytic sets defined by functions from a subalgebra closed under differentiation. Namely, the closure and the frontier of such a set (inside the domain of definition of the functions) can be defined as semianalytic sets within the same subalgebra. Also, there exists a stratification of such a set, with the strata defined by functions from the same subalgebra.

\section{Application to Pfaffian functions.}

When the functions in the original expression are Pfaffian (see [17]), the corresponding classes of semianalytic and subanalytic sets will be called semi-Pfaffian and sub-Pfaffian, respectively. Our method implies that the complement of a sub-Pfaffian set is sub-Pfaffian, with the defining functions from the algebra generated over polynomials by the Pfaffian functions from the original expression. The constructiveness of our techniques, combined with effective bounds on finiteness properties for Pfaffian functions given in [17] and [10], allows one to effectively estimate complexity of the existential expression, in terms of complexity of the original expression and of the Pfaffian functions involved. For semiand sub-Pfaffian sets, complexity of closure, frontier, and stratification can be effectively estimated. The detailed estimates will appear in a separate paper. One step in this direction, an estimate for the complexity of a weak stratification of a semi-Pfaffian set, has appeared in [11].

One important class of Pfaffian functions are the sparse polynomials, or fewnomials [17]. Outside coordinate hyperplanes, they can be considered as Pfaffian functions of complexity depending only on the number of non-zero monomials, independent of their 
degrees. Effective estimates on the complexity of quantifier simplification for Pfaffian expressions yield the corresponding estimates for sparse polynomials outside coordinate hyperplanes.

For Pfaffian functions in an unbounded domain or at the boundary of their domain of definition (e.g., for sparse polynomials at the coordinate hyperplanes) most of the finiteness properties are still true, and our method can be extended to this case, except for Lemma 1 below (describing the frontier of a semianalytic set) which involves Taylor expansions. Thus the problem of construction of an equivalent existential formula in this case is equivalent to a positive answer to the following question:

Consider $\mathbf{R}^{n}$ as a subset of $\mathbf{R} \mathbf{P}^{n}$, with $\mathbf{R} \mathbf{P}^{n-1}$ identified with the points at infinity. For a semi-Pfaffian set $X \subset \mathbf{R}^{n}$, its frontier at infinity is defined as $\bar{X} \cap \mathbf{R} \mathbf{P}^{n-1}$. Does the frontier at infinity of a $k$-dimensional semi-Pfaffian set belong to a "sub-Pfaffian" subset of dimension smaller than $k$ ? (Here "sub-Pfaffian" is to be understood as a projection of a semi-Pfaffian set with no compactness conditions.)

At present, the only known results in this direction are Wilkie's theorem [26] (see also [7]) on model completeness for expressions with exponential polynomials in an unbounded domain, and the theorem of Cano, Moussu and Lion [3] on the frontier of a Pfaffian hypersurface.

\section{Definitions and the main results.}

Definition 1. Let $\Phi=\left\{\phi_{j}\right\}$ be a set of real analytic functions $\phi_{j}$ defined and analytic in a neighborhood of the closed unit cube $\mathbf{I}^{n_{j}} \subset \mathbf{R}^{n_{j}}$ (where $\mathbf{I}=[0,1]$ ). For every $n \geq 0$, we define $A_{n}=A_{n}(\Phi)$ as the minimal set of functions with the following properties:

1. The constants 0 and 1 and a coordinate function $x_{1}$ on $\mathbf{R}$ belong to $A_{1}$.

2. $\phi_{j} \in A_{n_{j}}$, for each $j$.

3. If $\phi, \phi^{\prime} \in A_{n}$ then $\phi \pm \phi^{\prime} \in A_{n}$ and $\phi \cdot \phi^{\prime} \in A_{n}$.

4. If $\phi\left(x_{1}, \ldots, x_{n}\right) \in A_{n}$ then $\phi\left(x_{i(1)}, \ldots, x_{i(n)}\right) \in A_{n+m}$, for any mapping $i$ : $\{1, \ldots, n\} \rightarrow\{1, \ldots, m+n\}$.

5. If $\phi(x) \in A_{n}$ then $\partial \phi(x) / \partial x_{\nu} \in A_{n}$, for $\nu=1, \ldots, n$.

Definition 2. A subset $X \subset \mathbf{I}^{n}$ is called $\Phi$-semianalytic if it is a finite union of sets of the form

$$
\left\{x: f_{i}(x)=0, \text { for } i=1, \ldots, I ; \quad g_{j}(x)>0, \text { for } j=1, \ldots, J\right\}
$$

where the $f_{i}$ and $g_{j}$ are analytic functions from $A_{n}(\Phi)$. A subset $Y \subset \mathbf{I}^{n}$ is called $\Phi$ subanalytic if it is an image of the projection to $\mathbf{R}^{n}$ of a $\Phi$-semianalytic subset $X \subset \mathbf{I}^{m+n}$. 
Remark. Subanalytic sets are usually defined as images of semianalytic sets under relatively proper analytic maps. However, the definition given above is essentially equivalent to this formally more general definition.

Definition 3. For a set $X \subset \mathbf{I}^{n}$, let $\bar{X}$ denote its closure, $\tilde{X}=\mathbf{I}^{n} \backslash X$ its complement in $\mathbf{I}^{n}$, and $\partial X=\bar{X} \backslash X$ its frontier. A semianalytic set $X \subset \mathbf{R}^{n}$ is non-singular of dimension $k$ at a point $x_{0} \in X$ if there exist real analytic functions $h_{1}(x), \ldots, h_{n-k}(x)$ defined in an open set $U \ni x_{0}$ such that $d h_{1} \wedge \ldots \wedge d h_{n-k} \neq 0$ at $x_{0}$ and $X \cap U=\left\{x \in U: h_{1}(x)=\right.$ $\left.\ldots=h_{n-k}(x)=0\right\}$. A semianalytic set (1) is effectively non-singular if the functions $h_{1}, \ldots, h_{n-k}$ can be chosen from the set $f_{i}$. The dimension $\operatorname{dim} X$ of a set $X$ is defined as the maximum of its dimensions at non-singular points.

To simplify the notation, let $f, g$ denote the set of functions $f_{i}$ and $g_{j}$, respectively. We write " $f=0$ " instead of " $f_{i}=0$, for all $i$," etc. We also define $|f(x)|=\max _{i}\left|f_{i}(x)\right|$ and $g_{\min }(x)=\min _{j} g_{j}(x)$.

Theorem 1. Let $Y$ be a $\Phi$-subanalytic subset of $\mathbf{I}^{n}$. Then $\tilde{Y}=\mathbf{I}^{n} \backslash Y$ is $\Phi$-subanalytic.

Corollary. Every expression constructed from equalities and inequalities between functions from the set $\Phi$, arithmetic operations,,+- , and $\times$, logical operations $\wedge$ and $\vee$, existential and universal quantifiers, is equivalent to an existential expression with functions from $A_{N}(\Phi)$, for some $N$, provided that only values in $[0,1]$ are allowed for all variables.

This is an easy consequence of Theorem 1 .

The proof of Theorem 1 will be given in section 5, based on several properties of $\Phi$-semianalytic sets derived in section 4 . As a by-product of the induction procedure in the proof of Theorem 1, we obtain the following additional property of $\Phi$-subanalytic sets:

Theorem 2. Each $\Phi$-subanalytic set $Y \subset \mathbf{I}^{n}$ can be represented as a finite disjoint union of $\Phi$-subanalytic subsets $Y_{i}$ so that each $Y_{i}$ is a cell homeomorphic to an open $k_{i}$ dimensional cube. More precisely, for each $Y_{i}$ there exists a linear transformation with integer coefficients to a coordinate system $y_{1}, \ldots, y_{k_{i}}, y_{k_{i}+1}, \ldots, y_{n}$ such that

$$
\begin{gathered}
Y_{i}=\left\{a_{j}\left(y_{1}, \ldots, y_{j-1}\right)<y_{j}<b_{j}\left(y_{1}, \ldots, y_{j-1}\right), \text { for } 1 \leq j \leq k_{i} ;\right. \\
\left.y_{j}=c_{j}\left(y_{1}, \ldots, y_{k_{i}}\right), \text { for } j=k_{i}+1, \ldots, n\right\} .
\end{gathered}
$$

Here $a_{j}$ and $b_{j}$, for $1 \leq j \leq k_{i}$, are analytic functions of $y_{1}, \ldots, y_{j-1}$ with $\Phi$-subanalytic graphs, defined in an open domain

$$
\Omega_{j}=\left\{a_{\nu}\left(y_{1}, \ldots, y_{\nu-1}\right)<y_{\nu}<b_{\nu}\left(y_{1}, \ldots, y_{\nu-1}\right), \text { for } 1 \leq \nu \leq j\right\}
$$


and $a_{j}<b_{j}$ everywhere in $\Omega_{j}$. For for $k_{i} \leq j \leq n$, the functions $c_{j}$ are analytic in $\Omega_{k_{i}}$, with $\Phi$-subanalytic graphs.

\section{Preliminary lemmas.}

Lemma 1. Let $X=\{x: f(x)=0, g(x)>0\}$ where the functions $f_{i}$ and $g_{j}$ are analytic in $\mathbf{I}^{n}$. Then $\bar{X}$ and $\partial X$ are finite unions of semianalytic sets, each defined by a finite system of equalities and inequalities between polynomials in $x$, the functions $f_{i}, g_{j}$, and their partial derivatives.

Proof. Let us show first that there exist $c>0$ and $\kappa>0$ such that the closure of the set

$$
\left\{x^{\prime} \in X, g\left(x^{\prime}\right) \geq c\left|x^{\prime}-x\right|^{\kappa}\right\}
$$

contains $x$ if and only if $x \in \bar{X}$. This is obviously true when $x \in X$ or $x \notin \bar{X}$. To prove this for $x \in \partial X$, we consider a set

$$
A=\left\{(\epsilon, \delta): \exists x, D(x, \epsilon / 4) \neq \emptyset, \max _{x^{\prime} \in D(x, \epsilon)} g_{\min }\left(x^{\prime}\right)=\delta\right\} .
$$

Here $D(x, \epsilon)=\left\{x^{\prime} \in X,\left|x^{\prime}-x\right| \leq \epsilon\right\}$ is a ball in $X$ of radius $\epsilon$, centered at $x$. Due to [8], $A$ is a subanalytic subset in $\mathbf{R}^{2}$ (actually, a semianalytic subset, as every subanalytic subset in $\mathbf{R}^{2}$ is semianalytic according to [20]). We claim that $\bar{A}$ does not contain any points $(\epsilon, 0)$ with $\epsilon>0$. Otherwise there exist sequences $\left\{x_{\nu}\right\}$ in $\mathbf{I}^{n}$ and $\epsilon_{\nu} \rightarrow \epsilon>0$ such that $D\left(x_{\nu}, \epsilon_{\nu} / 4\right) \neq \emptyset$, and

$$
\max _{x^{\prime} \in D\left(x_{\nu}, \epsilon_{\nu}\right)} g_{\min }\left(x^{\prime}\right) \rightarrow 0 \text { as } \nu \rightarrow \infty
$$

The sequence $\left\{x_{\nu}\right\}$ has an accumulation point $x_{0} \in \mathbf{I}^{n}$. Due to the properties of the sequence $\left\{x_{\nu}\right\}$, we have $D\left(x_{0}, \epsilon / 2\right) \neq \emptyset$ and $g_{\min }\left(x^{\prime}\right) \leq 0$ on $D\left(x_{0}, \epsilon / 2\right)$, which is impossible.

Due to the "curve lemma" [20], there exist $c_{0}>0$ and $\kappa>0$ such that

$$
\delta \geq c_{0} \epsilon^{\kappa}+o\left(\epsilon^{\kappa}\right) \text { when }(\epsilon, \delta) \in A \text {, for small } \epsilon>0 .
$$

We want to show that, for any $c \leq c_{0}$ and $x \in \partial X$, the closure of the set (3) contains $x$. Let $\left\{x_{\nu}\right\}$ be a sequence in $X$ converging to $x$. Let $\epsilon_{\nu}=\left|x-x_{\nu}\right|$ and $\delta_{\nu}=\max _{x^{\prime} \in D\left(x, \epsilon_{\nu}\right)} g_{\min }\left(x^{\prime}\right)$, so that $\left(\epsilon_{\nu}, \delta_{\nu}\right) \in A$. Take $x_{\nu}^{\prime} \in D\left(x, \epsilon_{\nu}\right)$ with $g_{\min }\left(x_{\nu}^{\prime}\right)=\delta_{\nu}$, so $g_{\min }\left(x_{\nu}^{\prime}\right) \geq c_{0}\left(\epsilon_{\nu}\right)^{\kappa} \geq$ $c\left|x-x_{\nu}^{\prime}\right|^{\kappa}$, for large $\nu$. Hence $x_{\nu}^{\prime}$ belongs to the set (3), for large $\nu$, and $x_{\nu}^{\prime} \rightarrow x$ as $\nu \rightarrow \infty$.

We can suppose that $\kappa$ in (3) is integer. Due to (4) we can replace $g\left(x^{\prime}\right)$ in (3) by its Taylor expansion $\check{g}_{x}\left(x^{\prime}\right)$ of the order $\kappa$ at $x$, and the closure of (3) still contains $x$ if and only if $x \in \bar{X}$. This can be reformulated as follows: Let

$$
s_{x}=\left\{x^{\prime}: \check{g}_{x}\left(x^{\prime}\right)>0, \check{g}_{x}\left(x^{\prime}\right) \geq c\left|x^{\prime}-x\right|^{\kappa}\right\}
$$


Then the closure of the set

$$
\left\{x^{\prime} \in s_{x}, f\left(x^{\prime}\right)=0\right\}
$$

contains $x$ if and only if $x \in \bar{X}$.

We want to show that there exist $c^{\prime}>0$ and $\lambda>0$ such that the closure of the set

$$
\left\{x^{\prime} \in s_{x}:\left|f\left(x^{\prime}\right)\right| \leq c^{\prime}\left|x^{\prime}-x\right|^{\lambda}\right\}
$$

contains $x$ if and only if $x \in \bar{X}$. This is obviously true when $x \in \bar{X}$, or $f(x) \neq 0$, or $g_{\min }(x)<0$.

To prove this for $x \notin \bar{X}, f(x)=g_{\min }(x)=0$, we consider a subanalytic set

$$
B=\left\{(\epsilon, \delta): \exists x \in \mathbf{I}^{n}, \operatorname{dist}(x, X) \geq \epsilon, \max _{0 \leq \zeta \leq \epsilon} \min _{x^{\prime} \in s_{x},\left|x-x^{\prime}\right|=\zeta}\left|f\left(x^{\prime}\right)\right|=\delta\right\} .
$$

We claim that $\bar{B}$ does not contain any points $(\epsilon, 0)$ with $\epsilon>0$. Otherwise there exists a sequence $x_{\nu}$ in $\mathbf{I}^{n}$ with $\operatorname{dist}\left(x_{\nu}, X\right) \geq \epsilon_{\nu} \rightarrow \epsilon$ and

$$
\max _{0 \leq \zeta \leq \epsilon_{\nu}} \min _{x^{\prime} \in s_{x_{\nu}},\left|x_{\nu}-x^{\prime}\right|=\zeta}\left|f\left(x^{\prime}\right)\right| \rightarrow 0 \quad \text { as } \quad \nu \rightarrow \infty .
$$

Let $x_{0}$ be an accumulation point of the sequence $\left\{x_{\nu}\right\}$. We have $\operatorname{dist}\left(x_{0}, X\right) \geq \epsilon$, hence $x_{0} \notin \bar{X}$. Due to $(7)$, for every $\zeta \leq \epsilon$, there exists a point $x^{\prime} \in s_{x_{0}}$ with $\operatorname{dist}\left(x^{\prime}, x_{0}\right)=\zeta$ and $f\left(x^{\prime}\right)=0$. Hence the closure of the set (5) contains $x_{0}$, in contradiction to the definition of $c$ and $\kappa$.

Due to the "curve lemma" we have $\delta \geq c_{1} \epsilon^{\lambda}+o\left(\epsilon^{\lambda}\right)$, with $c_{1}>0$ and $\lambda>0$, when $(\epsilon, \delta) \in B, \epsilon \searrow 0$. Let us choose $c^{\prime}>c_{1}$. Let $x \notin \bar{X}$ and $f(x)=g_{\min }(x)=0$. Then, for small enough positive $\epsilon$,

$$
\left.\max _{0 \leq \zeta \leq \epsilon} \min _{x^{\prime} \in s_{x},\left|x-x^{\prime}\right|=\zeta}\left|f\left(x^{\prime}\right)\right| \geq c^{\prime} \epsilon^{\lambda}\right\}
$$

This implies that the closure of the set (6) does not contain $x$. We can suppose that $\lambda$ in (6) is integer.

Finally, we can replace $f\left(x^{\prime}\right)$ in (6) by its Taylor expansion $\check{f}_{x}\left(x^{\prime}\right)$ of order $\lambda$ at $x$, and the closure of the set

$$
S_{x}=\left\{x^{\prime} \in s_{x}:\left|\check{f}_{x}\left(x^{\prime}\right)\right| \leq c^{\prime}\left|x^{\prime}-x\right|^{\lambda}\right\}
$$

still contains $x$ if and only if $x \in \bar{X}$.

For a fixed $x$, the set $S_{x}$ is semialgebraic in $x^{\prime}$. According to the Tarski-Seidenberg theorem, the condition that the closure of $S_{x}$ contains $x$ is equivalent to a semialgebraic 
condition on coefficients of polynomials defining $S_{x}$, which are partial derivatives at $x$ of $f_{i}$, of order not greater than $\lambda$, and of $g_{j}$, of order not greater than $\kappa$.

This proves Lemma 1 for $\bar{X}$. The statement for $\partial X$ readily follows.

Lemma 2. Let $X$ be a semianalytic subset of $\mathbf{I}^{n}$. Then $X$ is a finite disjoint union of non-singular manifolds $X_{\alpha}$ such that each $X_{\alpha}$ is defined by a system of equalities and inequalities

$$
f_{\alpha \mu}(x)=0, \quad g_{\alpha \nu}(x)>0
$$

where the functions $f_{\alpha \mu}$ belong to the algebra generated by $f_{i}$ and their partial derivatives, the functions $g_{\alpha \nu}$ belong to the algebra generated by $f_{i}, g_{j}$ and the partial derivatives of $f_{i}$, and each set $X_{\alpha}$ with the presentation (8) is effectively non-singular.

Proof. We can suppose that $X$ belongs to the interior of $\mathbf{I}^{n}$. If this is not so, we can deal with the part of $X$ at the boundary of $\mathbf{I}^{n}$ separately, as it is a union of subsets of cubes of smaller dimensions. Also, we can suppose that $X$ is defined by equalities and inequalities as in (4), since any semianalytic set can be represented as a disjoint union of sets (4). The case $\operatorname{dim} X=n$ is trivial. Suppose that $\operatorname{dim} X<n$. Then there exists a function $h \not \equiv 0$ in the set $\left\{f_{i}\right\}$. Let $\nu$ be the minimal order of $h$ at the points of $X$ i.e., the minimal, over $x \in X$, power of non-zero terms in the Taylor expansion of $h$ at $x$. Then there exists a partial derivative

$$
s_{1}=\frac{\partial^{\nu} h}{\partial x_{i_{1}} \cdots \partial x_{i_{\nu}}}
$$

of $h$ of the order $\nu$ such that $Y_{1}=\left\{x \in X: s_{1}(x) \neq 0\right\}$ is a non-empty subset of $X$. Let

$$
h_{1}=\frac{\partial^{\nu-1} h}{\partial x_{i_{2}} \cdots \partial x_{i_{\nu}}} .
$$

Then $h_{1}=0$ and $\partial h_{1} / \partial x_{i_{1}}=s_{1} \neq 0$ at every point of $Y_{1}$. Let $X^{\prime}=\left\{x \in X: s_{1}(x)=0\right\}$ and $U_{1}=\left\{x: h_{1}(x)=0, s_{1}(x) \neq 0, g(x)>0\right\}$. The set $X^{\prime}$ is a proper subset of $X$ (possibly empty) and the set $U_{1}$ is non-singular of dimension $n-1$.

If $X^{\prime}$ is not empty, we proceed with $X^{\prime}$ in the same way as with $X$ i.e., define the partial derivative of $h$ that does not vanish identically on $X^{\prime}$, and so on.

With $Y_{1}$, there are two possibilities. If $Y_{1}$ is an open subset of $U_{1}$, we can set $X_{1}=Y_{1}$, adding $h_{1}$ to the functions $f_{i}$ and $s_{1}$ to the functions $g_{j}$ (strictly speaking, $Y_{1}$ in this case represents two effectively non-singular subsets of $X$, corresponding to $s_{1}>0$ and $s_{1}<0$ ).

Otherwise there exists a function $h$ in the set $\left\{f_{i}\right\}$ which does not vanish identically on $U_{1}$ in the neighborhood of $Y_{1}$. Let $\nu$ be the minimal order of $h_{U_{1}}$ at the points of $Y_{1}$. 
For $j \neq i_{1}$, the set of differential operators (derivatives along $U_{1}$ )

$$
\partial_{j}=\frac{\partial h_{1}}{\partial x_{j}} \frac{\partial}{\partial x_{i_{1}}}-\frac{\partial h_{1}}{\partial x_{i_{1}}} \frac{\partial}{\partial x_{j}}
$$

generate the tangent space to $U_{1}$ at every point. Hence there exists a partial derivative $s_{2}=\partial_{j_{1}} \cdots \partial_{j_{\nu}} h$ such that $Y_{2}=\left\{x \in Y_{1}: s_{2}(x) \neq 0\right\}$ is non-empty. At the same time, $h_{2}=\partial_{j_{2}} \cdots \partial_{j_{\nu}} h$ vanishes on $U_{1}$ in the neighborhood of every point of $Y_{2}$.

Let $i_{2}=j_{1}, X^{\prime \prime}=\left\{x \in Y_{1}: s_{2}(x)=0\right\}$, and $U_{2}=\left\{x \in U_{1}: h_{2}(x)=0, s_{2}(x) \neq 0\right\}$. Then $X^{\prime \prime}$ is a proper subset of $Y_{1},\left.h_{2}\right|_{U_{2}} \equiv 0$, and $\partial_{i_{2}} h_{2}=s_{2} \neq 0$ at every point of $U_{2}$. In particular, $U_{2}$ is non-singular of dimension $n-2$.

We proceed with $X^{\prime \prime}$ in the same way as with $Y_{1}$, i.e., define the partial derivative of $h$ along $U_{1}$ that does not vanish identically on $X^{\prime \prime}$, and so on.

For $Y_{2}=X \cap U_{2}$, there are two possibilities: either it is an open subset of $U_{2}$, or there exists a function $h$ in the set $\left\{f_{i}\right\}$ which does not vanish identically on $U_{2}$ in the neighborhood of $Y_{2}$. In the first case, $Y_{2}$ is effectively non-singular of dimension $n-2$, with $h_{1}$ and $h_{2}$ added to the list of the functions $f_{i}$ and $s_{1}$ and $s_{2}$ added to the list of the functions $g_{j}$. In the second case, we consider derivatives along $U_{2}$ :

$$
\partial_{j}=\left|\begin{array}{ll}
\frac{\partial h_{1}}{\partial x_{i_{2}}} & \frac{\partial h_{1}}{\partial x_{j}} \\
\frac{\partial h_{2}}{\partial x_{i_{2}}} & \frac{\partial h_{2}}{\partial x_{j}}
\end{array}\right| \frac{\partial}{\partial x_{i_{1}}}-\left|\begin{array}{cc}
\frac{\partial h_{1}}{\partial x_{i_{1}}} & \frac{\partial h_{1}}{\partial x_{j}} \\
\frac{\partial h_{2}}{\partial x_{i_{1}}} & \frac{\partial h_{2}}{\partial x_{j}}
\end{array}\right| \frac{\partial}{\partial x_{i_{2}}}+\left|\begin{array}{ll}
\frac{\partial h_{1}}{\partial x_{1}} & \frac{\partial h_{1}}{\partial x_{i_{2}}} \\
\frac{\partial h_{2}}{\partial x_{i_{1}}} & \frac{\partial h_{2}}{\partial x_{i_{2}}}
\end{array}\right| \frac{\partial}{\partial x_{j}},
$$

for $j \neq i_{1}, i_{2}$. We define $s_{3}$ as a partial derivative of $h$ along $U_{2}$ (i.e., a composition of the operators $\partial_{j}$ ) of the minimal order which does not vanish identically on $Y_{2}$, choose $h_{3}$ such that $s_{3}=\partial_{i_{3}} h_{3}$, set $U_{3}=\left\{x \in U_{2}: h_{3}(x)=0, s_{3}(x) \neq 0\right\}$, and so on.

We repeat this procedure until we finally represent $X$ as a union of effectively nonsingular subsets satisfying conditions of Lemma 2. Note that the procedure terminates after a finite number of steps, as at every step we either stop at an effectively non-singular (possibly empty) subset $Y$, or pass to a non-singular set $U$ of smaller dimension, or add an additional equation $s=0$ defining a proper subset of a semianalytic set. The latter operation cannot continue indefinitely because of the descending chain condition on zerosets of analytic functions on $I^{n}$.

Lemma 3. Let $X$ be a $\Phi$-semianalytic set in $\mathbf{R}^{m+n}$, and let $Y=\pi X \subset \mathbf{I}^{n}, d=\operatorname{dim} Y$. Then there exist finitely many $\Phi$-semianalytic subsets $X_{\nu}^{\prime}$ and $a \Phi$-subanalytic subset $V$ of $X$ such that $Y=\bigcup_{\nu} \pi X_{\nu}^{\prime} \bigcup \pi V$ and

a) $X_{\nu}^{\prime}$ is effectively non-singular, $\operatorname{dim} X_{\nu}^{\prime}=d$, and $\pi: X_{\nu}^{\prime} \rightarrow Y$ has rank $d$ at every point of $X_{\nu}^{\prime}$, for each $\nu$; 
b) $\operatorname{dim}(\pi V)<d$;

c) $X_{\mu}^{\prime} \cap X_{\nu}^{\prime}=\emptyset$, for $\mu \neq \nu$.

Proof. We proceed by induction on $k=\operatorname{dim} X$. According to Lemma 2, we can consider the case when $X$ is non-singular set defined by equalities and inequalities as in (4), and $\operatorname{rk}(\partial f / \partial x)=m+n-k$ at every point of $X$. Let $z=\left(x_{1}, \ldots, x_{m}\right)$ and $y=\left(x_{m+1}, \ldots, x_{m+n}\right)$ be coordinates in $\mathbf{R}^{m}$ and $\mathbf{R}^{n}$, and let

$$
V=X \cap\{\operatorname{rk}(\partial f / \partial z)<m-k+d\}
$$

be the critical set of $\left.\pi\right|_{X}$. Then $\operatorname{dim}(\pi V)<d$. Preimage $\pi^{-1} y$ of every $y \in Y \backslash \pi V$ is a smooth manifold of dimension $k-d$. We can suppose that its projection to a $(k-d)$ dimensional subspace in $\mathbf{R}^{m}$ is a diffeomorphism. Otherwise we can deal with a critical set of this projection separately. For $k>d$, this implies that every connected component of $\pi^{-1} y$ has a nonempty frontier. Let $g(x)=g_{1}(x) \cdots g_{J}(x)$. Then $g>0$ at every point of $X$ and $g \equiv 0$ at the frontier of $X$. Hence $g$ is not constant on every connected component of $\pi^{-1} y$ for every $y \in Y \backslash \pi V$. Let

$$
W=X \cap\{\operatorname{rk}(\partial(f, g) / \partial z)=m-k+d\} .
$$

Intersection of $W$ with $\pi^{-1} y$, for every $y \in Y \backslash \pi V$, coincides with the critical set of $g$ on $\pi^{-1} y$. Hence intersection of $W$ with every connected component $C$ of $\pi^{-1} y$ is a non-empty proper subset of $C$. In particular, $\pi W=Y$ and $\operatorname{dim} W<k$, q.e.d.

\section{Proof of Theorems 1 and 2.}

Let $X$ be a $\Phi$-semianalytic subset in $\mathbf{I}^{m+n}$ and $Y=\pi X \subset \mathbf{I}^{n}$. Let $x=\left(x_{1}, \ldots, x_{m+n}\right)$ and $y=\left(x_{m+1}, \ldots, x_{m+n}\right)$ be coordinates in $\mathbf{R}^{m+n}$ and $\mathbf{R}^{n}$, respectively.

We proceed by induction on $d=\operatorname{dim} Y$. As an inductive hypothesis, we suppose that, for any $\Phi$-subanalytic set $Y \subset \mathbf{I}^{n}$ of dimension $k<d$,

$\left(\mathrm{A}_{k}\right)$ the complement $\tilde{Y}$ of $Y$ is $\Phi$-subanalytic,

$\left(\mathrm{B}_{k}\right)$ the set $Y$ is a finite union of $\Phi$-subanalytic subsets of type (2),

$\left(\mathrm{C}_{k}\right)$ for $n=k+1$, there exists a closed $\Phi$-subanalytic set $Y^{\prime} \supset Y$ such that $\operatorname{dim} Y^{\prime} \leq k$ and each connected component of $\tilde{Y}^{\prime}$ is a $\Phi$-subanalytic set of type (2).

Let us show first that $\left(\mathrm{A}_{0}\right)-\left(\mathrm{C}_{0}\right)$ are valid. If $k=0$ then $Y$ is a finite set, and its connected components are isolated points. Let $N$ be the number of points in $Y$. For $n=1$, each point in $Y$ is a $\Phi$-subanalytic set of the form

$$
\left\{y \in Y: \exists y_{1} \in Y, \ldots, \exists y_{N-1} \in Y, y_{1}<\ldots<y_{\nu-1}<y<y_{\nu}<\ldots<y_{N-1}\right\}
$$


Here $\nu=1, \ldots, N$. For $n>1$, connected components of $Y$ are defined by (9) if we replace "<" by a lexicographic order in $\mathbf{R}^{n}$. This proves $\left(\mathrm{B}_{0}\right)$.

If $Y \subset \mathbf{R}^{1}$, connected components of $\tilde{Y}$ (intervals) are $\Phi$-subanalytic sets of the form

$$
\left\{y \in \mathbf{I}: \exists y_{1} \in Y, \ldots, \exists y_{N} \in Y, y_{1}<\ldots<y_{\nu-1}<y<y_{\nu}<\ldots<y_{N}\right\}
$$

Here $\nu=1, \ldots, N+1$. This proves $\left(\mathrm{C}_{0}\right)$.

For $n>1$, the set $\tilde{Y}$ is a union of $\Phi$-subanalytic sets defined by (10) if we replace "<" by a lexicographic order in $\mathbf{R}^{n}$. This proves $\left(A_{0}\right)$.

Now we want to prove $\left(\mathrm{A}_{d}\right)-\left(\mathrm{C}_{d}\right)$ when $d>0$. The idea is to consider $Y$ as a $d$ parametric family of 0-dimensional sets, and to apply the analogues of the formulas (9) and (10) to this family. As these formulas involve the number $N$ of points in $Y$, we have to ensure that the number of points in the sets of our $d$-parametric family does not depend on the parameters. To achieve this, we identify a $\Phi$-subanalytic set $Z \subset \mathbf{I}^{d}$, of dimension smaller than $d$, containing all possible values of the parameters where the number of points can change. Due to the inductive hypothesis, there exists a closed $\Phi$-subanalytic subset $Z^{\prime} \supset Z$, of dimension smaller than $d$, such that each connected component of $\tilde{Z}^{\prime}$ is a $d$ dimensional $\Phi$-subanalytic subset of type (2). The number of points remains constant over any connected component of $\tilde{Z}^{\prime}$, and the analogues of (9) and (10) can be applied, proving (B) and (C) and representing part of $\tilde{Y}$ over $\tilde{Z}^{\prime}$ as a $\Phi$-subanalytic set. Finally, the part of $Y$ that lies over $Z^{\prime}$ has dimension smaller than $d$ itself, and the inductive hypothesis can be applied to it to complete the proof of (A).

According to Lemma 3, we can consider the case when $X=\bigcup_{\nu} X_{\nu}^{\prime} \bigcup V$, a disjoint union, where $V$ is a $\Phi$-semianalytic set with $\operatorname{dim}(\pi V)<d$, the sets $X_{\nu}^{\prime}$ are effectively non-singular $d$-dimensional $\Phi$-semianalytic sets, and projection of $X_{\nu}^{\prime}$ into $\mathbf{R}^{n}$ has rank $d$ at every point of $X_{\nu}^{\prime}$, for each $\nu$.

Consider first the case $d=n$. Let $Z=\bigcup_{\nu} \pi \partial X_{\nu}^{\prime} \bigcup \pi V$. We have $\operatorname{dim} Z<d$. Due to the inductive hypothesis, we can find a closed $\Phi$-subanalytic subset $Z^{\prime} \supset Z$ of $\mathbf{R}^{d}$, with $\operatorname{dim} Z^{\prime}<d$, such that all connected components of $\tilde{Z}^{\prime}$ are $\Phi$-subanalytic sets of type (2). By the definition of $Z$, each connected component of $\tilde{Z}^{\prime}$ is either a subset of $Y$ or of $\tilde{Y}$. In particular, $\tilde{Z}^{\prime} \cap \tilde{Y}$ is a $\Phi$-subanalytic set, and $\tilde{Z}^{\prime} \cap Y$ is a finite disjoint union of $\Phi$-subanalytic subsets of type (2).

We have $\operatorname{dim}\left(Z^{\prime} \cap Y\right)<d$, hence $\widetilde{Z^{\prime} \cap Y}$ is $\Phi$-subanalytic, by the inductive hypothesis, and $Z^{\prime} \cap Y$ is a finite disjoint union of $\Phi$-subanalytic subsets of type (2). Thus $\tilde{Y}=$ $\left(\tilde{Z}^{\prime} \cap \tilde{Y}\right) \cup\left(Z^{\prime} \cap\left(\widetilde{Z^{\prime} \cap Y}\right)\right)$ is $\Phi$-subanalytic i.e., $\left(\mathrm{A}_{d}\right)$ is valid, and $Y=\left(\tilde{Z}^{\prime} \cap Y\right) \cup\left(Z^{\prime} \cap Y\right)$ 
is a finite disjoint union of $\Phi$-subanalytic subsets of type (2) i.e., $\left(\mathrm{B}_{d}\right)$ is valid. $\left(\mathrm{C}_{d}\right)$ is not applicable in the case $d=n$.

Let now $d<n$. According to the Koopman-Brown theorem [18, Theorem 5.I], for a generic direction $l$ in $\mathbf{R}^{n}$, projection $\rho$ of $\bar{Y}$ along $l$ to a $(n-1)$-dimensional subspace satisfies the following property:

$\left(^{*}\right)$ For each $y \in \bar{Y}$, the set $\rho^{-1}(\rho y)$ is finite.

Applying the Koopman-Brown theorem inductively, we can show that, for a generic $(n-d)$-dimensional linear subspace $L$ in $\mathbf{R}^{n}$, projection $\rho$ of $Y$ along $L$ to a $d$-dimensional subspace $\mathbf{R}^{d}$ of $\mathbf{R}^{n}$ satisfies (*).

As condition $\left(^{*}\right)$ is open in $L$, we can choose $L$ to be defined by linear equations with integer coefficients in $\mathbf{R}^{n}$. Subspace $\mathbf{R}^{d}$ can be chosen as a coordinate subspace of $\mathbf{R}^{n}$.

Let $S=\cup S_{\nu}$ be the union of the critical sets $S_{\nu}$ of projection $\rho \pi: X_{\nu}^{\prime} \rightarrow \mathbf{R}^{d}$. Then $S$ is a $\Phi$-semianalytic set. (Since each $X_{\nu}^{\prime}$ is effectively non-singular, $S_{\nu}$ is defined by zeroes of certain minors of the Jacobian matrix of functions defining $X_{\nu}^{\prime}$, in a properly chosen system of linear coordinates.) It follows from $\left(^{*}\right)$ that $\operatorname{dim} \rho \pi S<d$. Otherwise, the set of critical values of $\rho \pi$ would be $d$-dimensional, in contradiction to the Sard's theorem.

Let $Z=\rho \pi\left(\bigcup_{\nu} \partial X_{\nu}^{\prime} \cup V \bigcup S\right)$. We have $\operatorname{dim} Z<d$.

By the inductive hypothesis, there exists a closed $\Phi$-subanalytic set $Z^{\prime} \supset Z$ such that $\operatorname{dim} Z^{\prime}<d$, the set $\tilde{Z}^{\prime}$ is $\Phi$-subanalytic, and each connected component of $\tilde{Z}^{\prime}$ is a $d$-dimensional $\Phi$-subanalytic set of type (2).

By the definition of $Z$ and implicit functions theorem, the set $X^{\prime}=X \cap \pi^{-1} \rho^{-1} \tilde{Z}^{\prime}$ is a finite union of graphs of analytic functions over each connected component of $\tilde{Z}^{\prime}$. The same is true for $Y^{\prime}=Y \cap \pi^{-1} \rho^{-1} \tilde{Z}^{\prime}$.

By the condition $c$ ) of Lemma 3, the graphs for $X^{\prime}$ are all disjoint. However, after projection $\pi$ to $\mathbf{R}^{n}$, some of the different graphs for $Y^{\prime}$ can intersect along a subset $W$ of dimension smaller than $d$. To show that $W$ is a $\Phi$-subanalytic set, we consider a $\Phi$ subanalytic set

$$
W^{\prime}=\left\{y, \epsilon: y \in Y, \rho y \in \tilde{Z}^{\prime}, \epsilon \in \mathbf{R}^{1}, \exists y^{\prime} \in Y, y^{\prime} \neq y, \rho y^{\prime}=\rho y,,\left|y^{\prime}-y\right|<\epsilon\right\} .
$$

Then $W=\overline{W^{\prime}} \cap\{\epsilon=0\}$ is $\Phi$-subanalytic due to Lemma 1 .

Adding $\rho W$ to $Z$ and replacing $Z^{\prime}$ correspondingly, we can guarantee that the number $N$ of points in $Y$ over each connected component $C$ of $\tilde{Z}^{\prime}$ remains constant.

In this case, connected components $C_{\mu}$ of $Y \cap \rho^{-1} C$ are defined by the following existential expression, similar to (9):

$$
C_{\mu}=\left\{y \in Y: \rho(y) \in C, \exists y_{1} \in Y, \ldots, \exists y_{N-1} \in Y,\right.
$$




$$
\left.\rho^{\prime} y_{1}<\ldots<\rho^{\prime} y_{\mu-1}<\rho^{\prime} y<\rho^{\prime} y_{\mu}<\ldots<\rho^{\prime} y_{N-1}, \rho\left(y_{1}\right)=\ldots=\rho\left(y_{N-1}\right)=\rho(y)\right\} .
$$

Here $\mu=1, \ldots, N$ and " $<$ " is the order in the 1-dimensional space $L$. Obviously, $C_{\mu}$ are of type (2) as long as $C$ is of type (2).

As $\operatorname{dim} Y \cap \rho^{-1} Z^{\prime}<d$, this, together with the inductive hypothesis, proves $\left(\mathrm{B}_{d}\right)$.

For $n=d+1$, connected components $C_{\mu}$ of $\tilde{Y} \cap \rho^{-1} C$ are defined by the following existential expression, similar to (10):

$$
\begin{gathered}
C_{\mu}=\left\{y: \rho(y) \in C, \exists y_{1} \in Y, \ldots, \exists y_{N} \in Y,\right. \\
\left.y_{1}<\ldots<y_{\mu-1}<y<y_{\mu}<\ldots<y_{N}, \rho\left(y_{1}\right)=\ldots=\rho\left(y_{N}\right)=\rho(y)\right\} .
\end{gathered}
$$

Here $\mu=1, \ldots, N+1$.

Again, $C_{\mu}$ are of type (2) as long as $C$ is of type (2). Setting $Y^{\prime}=Y \cup \rho^{-1} Z^{\prime}$, we prove $\left(\mathrm{C}_{d}\right)$.

For $d<n-1$, the set $\tilde{Y} \cap \rho^{-1} C$ is the union of $C_{\mu}$ if " $<$ " is defined as a lexicographic order. As $Y_{0}=Y \cap \rho^{-1} Z^{\prime}$ has dimension smaller than $d$, the set $\tilde{Y}_{0}$ is $\Phi$-subanalytic due to inductive hypothesis. Hence $\tilde{Y}$, which is the union of $\tilde{Y}_{0} \cap \rho^{-1} Z^{\prime}$ and the sets $\tilde{Y} \cap \rho^{-1} C$ over all connected components $C$ of $\tilde{Z}^{\prime}$, is $\Phi$-subanalytic. This proves $\left(\mathrm{A}_{d}\right)$.

Acknowledgements. Part of this work was done when the author was visiting the Department of Mathematics at the University of Toronto, supported by NSERC research grant OGP0009070. This work was supported in part by the United States Army Research Office through the Army Center of Excellence for Symbolic Methods in Algorithmic Mathematics, Mathematical Sciences Institute of Cornell University (MSI), grant DAAL03-91C-0027. Author is grateful to P. Pedersen and D. Richardson for the kind invitation to participate in the MSI-sponsored Workshop on "Computational Real Algebra and Geometry" that gave a starting impulse to this work, and to E. Bierstone, P. Milman, and L. van den Dries for discussions.

\section{References}

[1] E. Bierstone and P.D. Milman. Semianalytic and subanalytic sets. Inst. Hautes Etudes Sci. Publ. Math., v.67, p.5-42 (1988)

[2] E. Bierstone and P.D. Milman. Geometric and differential properties of subanalytic sets. Bull. Amer. Math. Soc., v.25, p.385-393 (1991)

[3] F. Cano, J.M. Lion et R. Moussu. Frontière d'une hypersurface pfaffienne. Preprint Lab. Topologie Univ. Bourgogne, Dijon (1993) To appear in CRAS Paris. 
[4] J. Denef and L. van den Dries. P-adic and real subanalytic sets. Ann. Math., v.128, p.79-138 (1988)

[5] Z. Denkowska, S. Łojasiewicz, and J. Stasica Certaines propriétés élémentaires des ensembles sous-analytiques, Bull. Polish Acad. Sci. Math. v.27, p.529-536 (1979)

[6] L. van den Dries. A generalization of the Tarski-Seidenberg theorem, and some nondefinability results. Bull. Amer. Math. Soc., v.15, n.2, p.189-193 (1986)

[7] L. van den Dries, A. Macintyre, and D. Marker. The elementary theory of restricted analytic fields with exponentiation. Ann. Math., v.140, n.1, p.183-205 (1994)

[8] A. Gabrielov. Projections of semi-analytic sets. Functional Anal. Appl., v.2, n.4, p.282-291 (1968)

[9] A. Gabrielov. Mappings of analytic sets. Ph.D. Thesis, Dept. Mech. Math., Moscow State Univ., Moscow (1973)

[10] A. Gabrielov, Multiplicities of Pfaffian intersections and the Lojasiewicz inequality. Preprint Cornell MSI, Ithaca NY (1993)

[11] A. Gabrielov and N. Vorobjov, Complexity of stratifications of semi-Pfaffian sets. Preprint Cornell MSI, Ithaca NY (1993)

[12] R.M. Hardt. Stratification of real analytic mappings and images. Invent. Math., v.28, p.193-208 (1975)

[13] R.M. Hardt. Topological properties of subanalytic sets, Trans. Amer. Math. Soc., v.211, p.57-70 (1975)

[14] R.M. Hardt. Triangulations of subanalytic sets and proper light subanalytic maps. Invent. Math., v.38, p.207-217 (1977)

[15] R.M. Hardt. Stratifications via corank one projections. Proc. Sympos. Pure Math., v.40, part 1, Amer. Math. Soc., Providence RI, p.559-566 (1983)

[16] H. Hironaka. Subanalytic sets. Number Theory, Algebraic Geometry and Commutative Algebra (in honor of Y. Akizuki), Kinokunya, Tokyo, p.453-493 (1973)

[17] A.G. Khovanskii, Fewnomials. AMS Translation of mathematical monographs; v.88, AMS, Providence RI (1991) Russian original: Malochleny, Moscow (1987)

[18] B.O. Koopman and A.B. Brown. On the covering of analytic loci by complexes. Trans. Amer. Math. Soc., 34, n.2 (1932)

[19] S. Łojasiewicz. Triangulation of semi-analytic sets. Ann. Scu. Norm. di Pisa, v.18, p.449-474 (1964) 
[20] S. Łojasiewicz. Ensembles semi-analytiques. Lecture Notes, IHES, Bures-sur-Yvette (1965)

[21] W. Pawlucki. On relations among analytic functions and geometry of subanalytic sets. Bull. Polish Acad. Sci. Math., 37, p.117-125 (1989)

[22] A. Seidenberg. A new decision method for elementary algebra. Ann. Math., v.60, p.365-374 (1954)

[23] H.J. Sussmann. Real-analytic desingularization and subanalytic sets: an elementary approach. Trans. Amer. Math. Soc., 317, n.2, p.417-461 (1990)

[24] A. Tarski. A decision method for elementary algebra and geometry, Berkeley note (1951)

[25] B. Teissier. Sur la triangulation des morphismes sous-analytiques. Inst. Hautes Etudes Sci. Publ. Math., v.70, p.169-198 (1989)

[26] A.J. Wilkie, Some model completeness results for expansions of the real field, I: restricted Pfaffian functions, and II: the exponential function, to appear in Journal of the American Math. Soc. (1995) 